\title{
Design and Development of Wireless Communicating and Sensing Module for Medical Equipments
}

\author{
Shihua Cao ${ }^{1 *}$, Qihui Wang ${ }^{2}$, Lidong Wang ${ }^{3}$, Huixi Zhang ${ }^{4}$, Yuhai Wang ${ }^{5}$, \\ Manna Zhang ${ }^{6}$ \\ College of Qianjiang, Hangzhou Normal University, Hangzhou,310036, China \\ 1email:caoshihua@126.com, ${ }^{2}$ emai:wangqihui_xinyi@hotmail.com, ${ }^{3}$ email: violet_wld@163.com, \\ email:zhhuixi@126.com, ${ }^{5}$ email:5472728@qq.com, ${ }^{6}$ email: 1458367654@qq.com, \\ *email:caoshihua@126.com
}

Keywords: Wireless Transmission; Medical Equipment; Sensing; Monitoring

\begin{abstract}
In view of the problems of poor equipment mobility and limited transmission distance when the existing hospital medical equipments transmits data to the hospital information system through wired connection, a type of wireless communicating and sensing module for medical equipments was designed, which connects the hospital information system for existing medical equipments through wireless networking, and can greatly increase the data transmission distance, enhance the flexibility of utilization, and facilitate the remote monitoring and management of medical equipments.
\end{abstract}

\section{Introduction}

With the continuous development of hospital information system, more and more medical equipments are adopted in hospitals. At present, the vast majority of medical equipments transmit data to the external application systems or monitoring centers through serial port, USB and Ethernet cable connection. The way of wired connection will limit the mobility and flexibility of medical equipments. Especially, now the demands of hospital's clinical diagnosis, nursing and management for medical service mobile processing is increasing, so it is necessary to develop and design a wireless data transmission system without changing the existing medical equipments. At present, the domestic and foreign research still stays at the state of developing wireless data forwarding adapter in accordance with the medical device interface, and the current research can only fulfill the wireless data access function for medical equipments, but the mobile state and location information of medical equipments have not been involved. ${ }^{\mathbf{1 1}-3 \mathbf{1}}$ However, these functions are quite significant for the operation management and monitoring of medical equipments. This research will discuss the wireless data access, the movement state and position information monitoring of medical equipments.

\section{Wireless Data Sensing Module Based on ZigBee}

ZigBee technology is a kind of two-way wireless communication technology, which has such features as: short distance, low complexity, low power consumption, low speed and low cost. It is mainly used for data transmission between different kinds of electronic devices that have short distance transmission, low power consumption and low transmission rate, as well as the typical applications with periodical data, intermittent data and low response time data transmission. ${ }^{\text {(41 }}$ ZigBee works in $2.4 \mathrm{GHz}$ frequency band, and the highest data transfer rate is up to $250 \mathrm{kbit} / \mathrm{s}$ for the transmission distance ranging from 10 to 75 meters, and it can form 65000 wireless data 
transmission networks.

Module structure: The medical wireless data sensing module based on ZigBee is composed of data interface (232, 485, Ethernet), power module, motion detection module, routing inspection module, positioning module, ZigBee module and micro control unit (MCU), as shown in Figure 1. The main functions include medical equipment asset management, access of internet of things and real-time monitoring on medical equipments, to solve the problem that the data cannot be timely and effectively transmitted and presented after the medical equipments collect data of the patients, medical personnel and environment, so users can know the patient signs, effectively manage the equipments on real-time basis, and intervene the abnormal situation in time. The design of data transmission module adheres to the principle of max hardware compatibility, and its communication adopts ZigBee wireless access mode, to realize connection with existing medical equipments through 232/485/USB/ Ethernet.

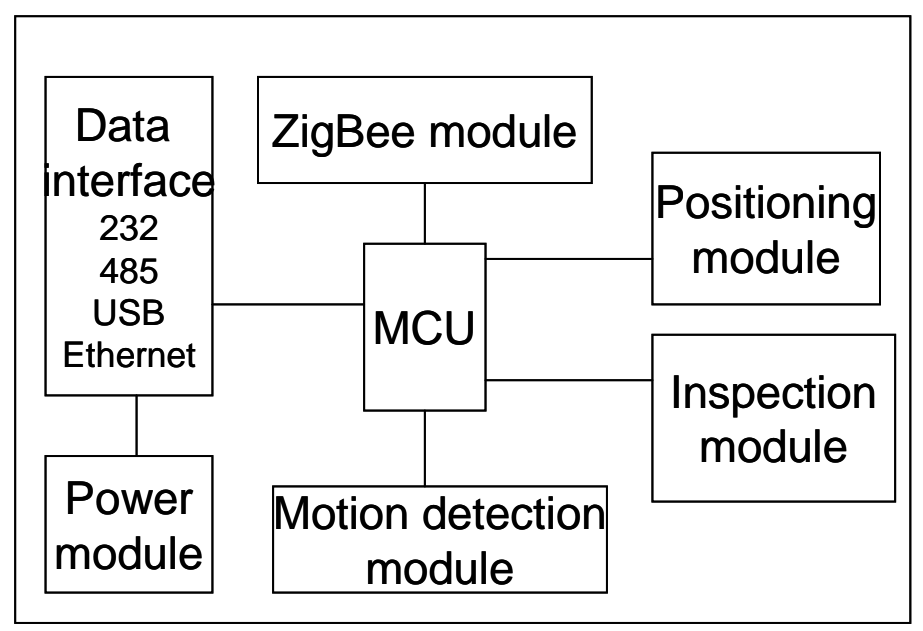

Figure 1 Wireless Data Transmission Module

The hardware design for data transmission module: the data transmission module adopts the most widely used Reduced Instruction Set Computer (RISC) microprocessor structure. The data transmission module can receive electric power from the medical equipments when it is connected with them, but it also has built-in chargeable 3.7V lithium battery inside the module. When the medical equipment works, the module can automatically charge the internal lithium battery through the charging chip. When the power from medical equipments is cut off, the internal lithium battery can guarantee the normal operation of the module. The three-axis acceleration sensor is used to detect the movement state of medical equipments, such as: stillness, motion and speed, etc. The positioning module is mainly composed of $125 \mathrm{~K}$ and $433 \mathrm{Mhz}$ high and low frequency RFID chips: the $433 \mathrm{Mhz}$ chip provides area or room positioning, and the $125 \mathrm{Khz}$ provides precise point positioning (1-2 $\mathrm{m}$ accuracy) [5]. The routing inspection module mainly provides records of regular inspection and maintenance operations for precious medical equipments. When the inspection personnel inspect and maintain the medical equipments, they will sense the inspected modules with inspection device, so as to record the inspection personnel, maintenance method, maintenance time and other data. The data transmission module transmits all the data of medical equipments to the background system through ZigBee module, and adopts ZigBee alliance certification to support ZigBee module of the standard ZigBee RPO protocol. 


\section{Data Communication Format and Software Design}

Wireless data transmission and sensing communication data format includes data head, mark bit, data length, user data, motion status, positioning data, inspection data, check bit and end mark, etc. as shown in Figure 2. The data transmission module can independently obtain real-time data from medical instruments and equipments, and then send the data to the data cloud in a timely manner through ZigBee network. The data transfer mechanism includes the response mechanism to make sure the data to be accurately received by data cloud. At the same time, with the data backup function of the module, when the data has been transferred successfully, the data can be saved to the Flash module inside the module for review and download by users at any time. In case of abnormal data transmission, the data will be temporarily saved to the internal module for resending later until the data is transmitted successfully. The data transmission module can be operated through internal software, to realize the seamless connection of modules between different ZigBee networks and enhancement of user experience.

\begin{tabular}{|l|l|l|l|l|l|l|l|l|}
\hline Head & Mark & Length & $\begin{array}{l}\text { User } \\
\text { Data }\end{array}$ & $\begin{array}{l}\text { Motion } \\
\text { status }\end{array}$ & $\begin{array}{l}\text { Positioning } \\
\text { Data }\end{array}$ & $\begin{array}{l}\text { Inspection } \\
\text { Data }\end{array}$ & CRC & End \\
\hline 8bit & 8bit & $8 \mathrm{bit}$ & $\mathrm{N}$ & $8 \mathrm{bit}$ & $8 \mathrm{bit}$ & $8 \mathrm{bit}$ & $8 \mathrm{bit}$ & $8 \mathrm{bit}$ \\
\hline
\end{tabular}

Figure 2 Wireless Data Transmission Module

\section{Experiment and results}

After the ZigBee based wireless data transmission and sensing module of medical equipments has been designed, installed and deployed, when the ZigBee transmission power is $0 \mathrm{dBm}$ and the indoor wireless data transmission distance of medical equipments is within the range of 30-50meters, the data receiving rate is $99.99 \%$; the outdoor transmission distance can reach 100 meters. By reasonably deploying ZigBee data receiving device, it can easily realize full coverage of wireless data reception on the entire floor and in the whole building. The real time positioning detection module and mobile detection module can collect the position and motion data of medical equipments at real time, through software operating and judgment, to display the position and motion state (falling, movement, moving upstairs or downstairs) of medical equipment at real time. as shown in Figure 3.

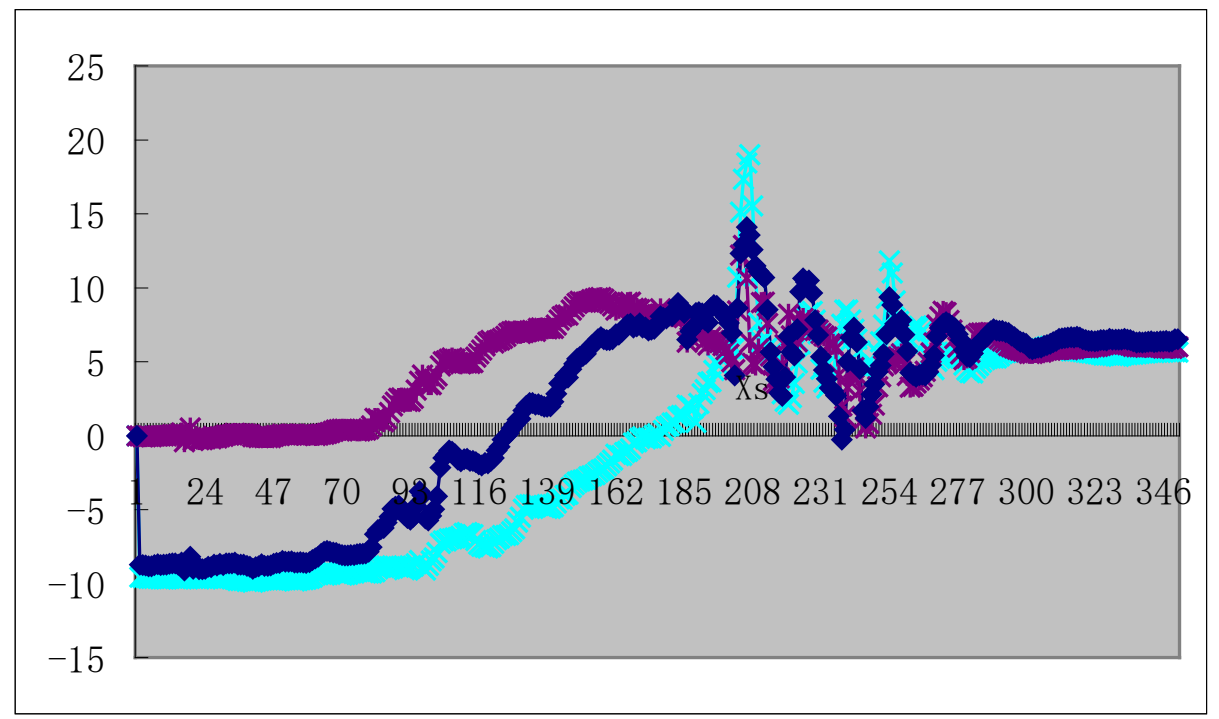

Figure 3. Data of motion detection module 
Through the implementation of the wireless data sensing module, the medical personnel and management personnel can easily realize remote and centralized monitoring on the operation data of medical equipments, and know the position and status information of medical equipments without staying with the medical device. In the past, if you want to find a medical device, usually you had to inquire several doctors, nurses or administrators, but now, you can quickly and accurately find the required medical equipments within 3-5 seconds by inquiring the software. This greatly improves the work efficiency of medical personnel and management personnel. Therefore, we can realize innovation and promotion of working mode of front-line clinical health care personnel, the optimization and standardization of business process, and the planning and reconstruction of management path; at the same time, on the basis of constructing clinical information standardized cloud center platform, we can further realize the collection, sharing, statistics, analysis, mining and utilization of electronic data. Through the application of wireless data transmission and sensing module of medical equipments, we can enhance clinical medical security and service quality, and completely record the electronic processes, so as to further realize the clinical treatment control and monitoring.

\section{Conclusion}

This paper discussed a Wireless Communicating and Sensing Module for Medical Equipments based Zigbee, analyzed it's working principle and the designing of the hardware and software module. Experiment shows that this module can provide the wireless data communication for the medical equipments and the motion and positioning status information, it improved the medical staffs working efficiency and the advanced management of the hospital.

\section{ACKNOWLEDGMENT}

This work is supported by the Natural Science foundation of Zhejiang Province,China(Grant No. LQ14F020008), Hangzhou Science and Technology Committee, China; (Grant No.20140633B34), Key disciplines project of Hangzhou Education Bureau, and Qian Jiang College, Hangzhou Normal University, China, 2015QJJL01, and Student Research and Innovation Program (Fresh talent plan) of Zhejiang Province(2014R421023).

\section{Reference:}

[1]Zheng Ye,Zhang Bohu,Design of data capturing and wireless transmission module based ARM7[J], Electrical technology, 2012,25(10):47-48.

[2]Yang Yong,Peng Hongbo,Chen Xiaocheng, Design and development of mobile wireless transmission system[J], China digital medical,2007.2(12):42.

[3]Chang Yongyu,Yang Ning. CDMA Wireless Positioning Technology and Application,Communication Technology, 2003,6 :1-5

[4] Zhang Hao,Zhao Qianchuan. Indoor Positioning System of Bluetooth Mobile Phone.Computer Application ,2007,31 (11):77-79.

[5]Yu Guancheng,Zhao Xiaodong and $\mathrm{Yu}$ Qian, super low power intelligent mobile electrocardiogram monitoring equipment[J],sensors and micro system. 2015,34(3):75-76

[6]Cao Shihua,,Zhao Fang, Research of application of LF wakeup technology in micro power active RFID system[J],micro computer system,2008,24(1):230-231 